\title{
Interference Model for Spectrum Sensing with Power Control
}

\author{
Yuandao Sun and Brian L. Mark \\ Dept. of Electrical and Computer Engineering \\ George Mason University, MS 1G5 \\ 4400 University Drive, Fairfax, VA \\ email: ysun12@gmu.edu; bmark@gmu.edu
}

\begin{abstract}
This paper analyzes the aggregate interference in a cognitive radio network. We consider a model in which the secondary transmitters are geographically distributed according to a Poisson point process in a wireless environment subject to shadowing noise and path loss. The secondary transmitters may transmit simultaneously and consequently incur an aggregate interference level on the primary system. We derive the moment generating function and the expectation of the aggregate interference for this model using properties of the Poisson point process. Based on the interference analysis, a scheme is proposed to estimate the maximum transmission power for each of the active secondary transmitters so as to satisfy a constraint on the aggregate interference. Our numerical results show a significant gain in the achievable capacity for the secondary system relative to an earlier scheme in which only a single secondary transmitter was permitted to transmit at any given time. ${ }^{1}$
\end{abstract}

Index Terms-Cognitive radio, Spectrum sensing, Poisson point process, Interference, Power Control

\section{INTRODUCTION}

In wireless networks, the available spectrum resource has become more and more scarce due to the explosive development of wireless communications. However, spectrum measurement studies have shown that significant portions of wireless spectrum are highly underutilized [1], [2]. To exploit the unused spectrum resource, dynamic or opportunistic spectrum access has been proposed which permits communication between the secondary users (unlicensed users) without sacrificing the communication performance of the primary users (licensed users). This can be achieved by cognitive radio technology, which is capable of sensing the available spectrum and dynamically tuning to different frequency channels to access it.

There has been much recent research activity on cognitive radios and opportunistic spectrum access. We focus here on spectrum sensing in the spatial domain. A key parameter in spatial spectrum sensing is the maximum interference-free transmit power (MIFTP), which is defined as the maximum power at which a given secondary transmitter can transmit without causing harmful interference to any of the primary receivers. In [3], the MIFTP for the secondary transmitter is estimated based on signal strength measurements taken by a group of secondary nodes. However, this is done under the

\footnotetext{
${ }^{1}$ This work was supported in part by the U.S. National Science Foundation under Grant CCF-0916568.
}

assumption that the secondary transmitters have the knowledge of their exact locations, and can exchange their observation and transmission decisions with negligible delay. Further, it is assumed that at most one secondary transmitter can transmit at a given time. In practice, due to the uncertainty in the number and locations of secondary nodes as well as channel fading, shadowing, and other uncertain environment-dependent conditions, the communication performance of the primary users may be seriously affected by the aggregate interference generated by multiple secondary users transmitting simultaneously [4]. Thus, accurate modeling of the aggregate interference is crucial to designing a cognitive radio network and quantifying the effect of the interference on the performance of the primary system.

A mathematical framework for characterizing interference in a cognitive radio network was proposed in [5]. In this framework, the secondary transmitters are scattered in the Euclidean plane according to a Poisson point process and operate asynchronously in a wireless environment subject to path loss, shadowing, and multipath fading. Although the aggregate interference from the secondary transmitters and its impact on the performance of the primary transmitter are quantified, the paper does not address how to utilize this information to carry out power control. Several papers in the literature have addressed the issue of power control in a cognitive radio network, see, e.g., [6]-[8].

In this paper, we adopt the framework in [5] and develop a spatial spectrum sensing model in which the secondary transmitters are scattered in the Euclidean plane according to a spatial Poisson point process. All transmissions are assumed to be omnidirectional and the signal propagation follows a lognormal shadowing model, which includes a path loss component and shadowing. We consider a single primary transmitter located in the center of the plane, together with the population of secondary transmitters. This models a cognitive radio network in which the primary transmitter population is relatively sparse. Based on this model, the aggregate interference is quantified. Furthermore, a power control scheme for the secondary transmitters, taking into account the aggregate interference, is proposed. In particular, we analyze the following scenarios:

1) Fixed secondary transmission power: In this scenario, the secondary users employ a simple Listen-Before-Talk dynamic spectrum access scheme with fixed transmis- 
sion power during the talk phase (see, e.g., [9], [10]). Using the distribution of the secondary transmitters and the propagation loss model, we derive the moment generating function and expectation of the aggregate interference.

2) Secondary transmitters with power control: This scenario takes the tolerable interference threshold of the primary receiver into account. A maximum transmit power for each of the active secondary transmitters is determined by taking into account the aggregate interference caused by their simultaneous transmissions.

The remainder of the paper is organized as follows. Section II describes the system model for spatial spectrum sensing. Section III develops an approach to derive an expression for the moment generating function and the expectation of the aggregate interference for this model under the assumption of fixed secondary transmission power. Section IV proposes a power control scheme for secondary transmitters based on the aggregate interference model. Section V presents simulation results. Finally, the paper is concluded in Section VI.

\section{System ModeL}

We consider a spatial spectrum sensing model with a single primary transmitter node $p$, one primary receiver node $v$ (or victim node) and a population of secondary transmitters distributed within an area in the Euclidean plane. The point process of secondary transmitters is assumed to form a realization of a homogeneous Poisson point process with intensity $\Lambda$. Each secondary transmitter is active with probability $\alpha$, independent of the other secondary transmitters. Using the thinning property of the Poisson point process (see, e.g., [11]), the active secondary transmitters form a realization of a homogeneous Poisson point process with intensity $\lambda=\alpha \Lambda$. For an area of interest $\mathbb{A}$, let $\Phi(\mathbb{A})$ denote the number of active secondary transmitters in $\mathbb{A}$ and let $|\mathbb{A}|$ denote the physical area corresponding to $\mathbb{A}$. Then the probability distribution of $\Phi(\mathbb{A})$ is given by

$$
P\{\Phi(\mathbb{A})=k\}=e^{-\lambda|\mathbb{A}|} \frac{(\lambda|\mathbb{A}|)^{k}}{k !}, k=0,1,2 \ldots
$$

Let $\mathcal{A}=\{1, \ldots, \Phi(\mathbb{A})\}$ denote the index set of active secondary transmitters and let $a_{i}$ denote the $i$ th secondary transmitter, $i \in \mathcal{A}$. The system scenario is illustrated in Fig. 1. Here, nodes $v_{1}, v_{2}$, and $v_{3}$, represent hypothetical victim nodes that lie closest to active secondary transmitters $a_{1}, a_{2}$, and $a_{3}$, respectively, within the coverage area of $p$.

We assume that all transmissions are omnidirectional and that signal propagation is governed by a lognormal shadowing model [12, (2.4.15)]. Hence, the propagation loss in $\mathrm{dB}$ between two nodes $i$ and $j$ can be expressed as

$$
L_{i, j}=g\left(d_{i, j}, \kappa\right)+W \quad[\mathrm{~dB}],
$$

where the function $g(d, \kappa)$ represents the path loss component, with $\kappa$ denoting the path loss factor [13]. For simplicity we assume that $g(d, \kappa)=10 \kappa \log _{10} d$ and $\kappa$ is given, so it can be denoted by $g(d)$. The shadowing noise $W$ is typically modeled as a zero-mean white Gaussian noise process with variance $\sigma_{w}^{2}$, which is independent of the path loss [14]. We denote the Gaussian distribution with mean $\mu$ and variance $\sigma^{2}$ by $N\left(\mu, \sigma^{2}\right)$. Thus, $W \sim N\left(0, \sigma_{w}^{2}\right)$. Hence, the received power at node $v$ due to node $p$ is given by

$$
R_{v}=s_{p}-L_{p, v}=s_{p}-g\left(d_{p, v}\right)+W \quad[\mathrm{dBm}],
$$

where $s_{p}$ is the transmitted power of node $p$. Similarly, the received power at node $v$ from node $a$, where node $a$ is any node chosen from $\mathcal{A}=\{1, \ldots, \Phi(\mathbb{A})\}$, is given by

$$
I_{v}=s_{a}-L_{a, v}=s_{a}-g\left(d_{a, v}\right)+W \quad[\mathrm{dBm}],
$$

where $s_{a}$ is the transmitted power of node a.

The outage probability of a victim node $v$ with respect to the transmitter $p$, is the probability that the received power $R_{v}$ from node $p$ falls below a detection threshold $r_{\min }[\mathrm{dBm}]$ when $p$ is transmitting:

$$
P_{\text {out }}(p, v) \triangleq P\left\{R_{v}<r_{\text {min }}\right\} .
$$

In general, $r_{\min }$ is determined by the primary receiver's structure, noise statistics, and quality of service (QoS) requirements. The coverage distance is the maximum distance between the node $p$ and any potential victim node $v$ such that the outage probability does not exceed a threshold $\varepsilon_{c o v}>0$ :

$$
\begin{aligned}
d_{\text {cov }}(p) & \triangleq \max \left\{d_{p, v}: P_{\text {out }}(p, v) \leqslant \varepsilon_{\text {cov }}\right\} \\
& =g^{-1}\left(s_{p}-r_{\text {min }}+\sigma Q^{-1}\left(1-\varepsilon_{\text {cov }}\right)\right),
\end{aligned}
$$

where $g^{-1}(\cdot)$ denotes the inverse of $g(\cdot)$ and $Q(x) \triangleq$ $\frac{1}{\sqrt{2 \pi}} \int_{x}^{\infty} e^{-\frac{t^{2}}{2}} d t$ denotes the standard Q-function.

The interference probability with respect to a given victim node $v$ is the probability that $I_{v}$ exceeds an interference tolerance threshold $I_{\max }[\mathrm{dBm}]$ when node $a$ is transmitting. This threshold can be set to meet the primary system's interference tolerance policy as follows:

$$
P_{\text {int }}(a, v) \triangleq P\left\{I_{v} \geqslant I_{\max }\right\} .
$$

Under the shadowing model, the interference probability is given by

$$
P_{i n t}(a, v)=Q\left(\frac{I_{\max }-s_{a}+g\left(d_{a, v}\right)}{\sigma_{v a}}\right) .
$$

\section{Aggregate INTERFERENCE WITH FIXED TRANSMISSION POWER}

In this section, we analyze the aggregate interference for a cognitive radio network represented in a Euclidean plane. In the Euclidean plane, the norm for a position vector $z=$ $\left(z_{1}, z_{2}\right)$ is given by $\|z\|=\sqrt{z_{1}^{2}+z_{2}^{2}}$. The scenario is shown in Fig. 1, with the primary transmitter located in the center of the plane. We assume that all secondary transmitters employ a Listen-Before-Talk (LBT) scheme [9], [10] to dynamically access the available spectrum. In the LBT scheme, each secondary transmitter consists of two states: listen state and talk state. During the listen state, the secondary transmitter does not transmit any signal, but it estimates the power of the received signal from the primary transmitter to determine whether it can switch to the talk state to transmit a signal. For example, if the received power $R<\eta$, the secondary 


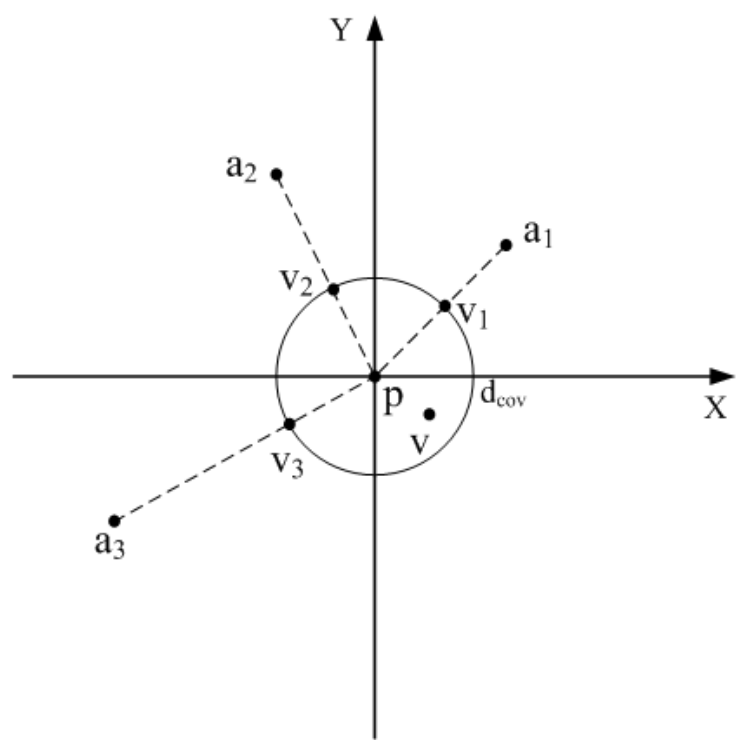

Fig. 1. Interference scenario.

transmitter would transition to the talk state; otherwise, it remains in the listen state. During the talk state, the secondary transmitter can transmit at a fixed power. After transmitting for a pre-specified maximum duration, it returns to the listen state to listen again. Compared with other models, which depend on the primary receivers to send out beacons to notify other nodes of their locations [5], the LBT model requires less energy and fewer bandwidth resources.

In the LBT scheme, each of the active secondary transmitters $a_{i}, i \in \mathcal{A}$, compares the received power from the primary transmitter to a deterministic threshold $\eta$. We introduce a function $U(x)$ to indicate whether a secondary transmitter located at position $x$ in the plane has the right to access the network:

$$
U(x)= \begin{cases}1, & s_{p}-g(\|x\|)+W_{p a}<\eta, \\ 0, & \text { otherwise, }\end{cases}
$$

where $W_{p a} \sim N\left(0, \sigma_{p a}^{2}\right)$ is the shadowing noise between the primary and secondary transmitters. If $U(x)=1$, a secondary transmitter located at position $x$ is sufficiently far away that it can transmit to its corresponding secondary receiver in the same spectrum band as the primary transmitter.

In this section, we assume that each secondary transmitter $a_{i}, i \in \mathcal{A}$, transmits with a fixed power $s_{a}$ if it has the right to access network. Let $x_{i}$ denote the position vector corresponding to the secondary transmitter $a_{i}, i \in \mathcal{A}$. The shadowing noise received at the victim node $v$ due to the transmission of secondary transmitter is given by $W_{v a} \sim$ $N\left(0, \sigma_{v a}^{2}\right)$. Hence, given the location of the primary receiver $x_{v}$, the aggregate interference from the secondary transmitters can be characterized as

$I=\sum_{i \in \mathcal{A}} U\left(x_{i}\right) \cdot 10^{\frac{s_{a}-g\left(\left\|x_{i}-x_{v}\right\|\right)+W_{v a}}{10}}=\sum_{i \in \mathcal{A}} U\left(x_{i}\right) h\left(x_{i}\right) 10^{\frac{W_{v a}}{10}}$,

where the summation is over the index set of the secondary transmitters $a_{i}, i \in \mathcal{A}$, and

$$
h(x)=10^{\frac{s_{a}-g\left(\left\|x-x_{v}\right\|\right)}{10}} .
$$

Here, the aggregate interference is given in linear scale. Given the location information $\Omega$ of all the secondary transmitters, the moment generating function (see, e.g., [15, Chap. 8]) of the aggregate interference can be derived as follows:

$$
\begin{aligned}
M_{I}(t) & =E\left[e^{t I}\right]=E\left[e^{t \sum_{i \in \mathcal{A}} U\left(x_{i}\right) h\left(x_{i}\right) 10^{\frac{W_{v a}}{10}}}\right] \\
& =\prod_{i \in \mathcal{A}} E\left[e^{t U\left(x_{i}\right) h\left(x_{i}\right) 10^{\frac{W v a}{10}}}\right] \\
& =E\left[\prod_{i \in \mathcal{A}} E\left[e^{t U\left(x_{i}\right) h\left(x_{i}\right) 10^{\frac{W_{v a}}{10}}} \mid \Omega\right]\right]
\end{aligned}
$$

Suppose $\left\{x_{i}\right\}$ is a Poisson point process with intensity measure $m(x) d x$. Using properties of the Laplace functional of the Poisson point process [11], [16], we can obtain the following equation for all nonnegative functions $v(x)$ satisfying $\int_{R^{2}}(1-v(x)) m(x) d x<\infty$ :

$$
E\left[\prod_{i \in \mathcal{A}} v\left(x_{i}\right)\right]=\exp \left\{-\int_{\mathbb{R}^{2}}(1-v(x)) m(x) d x\right\} .
$$

Let

$$
v(x)=E\left[e^{t U(x) h(x) 10^{\frac{W_{v a}}{10}}} \mid \Omega\right] .
$$

Applying (12) and (13) into (11), we can obtain the following alternative expression for $M_{I}(t)$ :

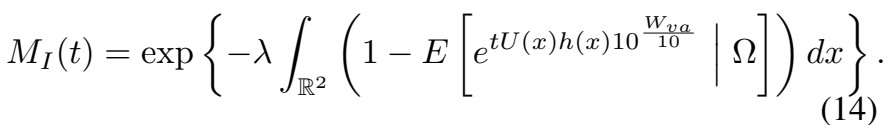

From the moment generating function, we can obtain the expectation of the aggregate interference. The shadowing noise variables $W_{p a}$ and $W_{v a}$ are independent of each other, and are independent of the locations of the secondary transmitters. For simplicity, $W_{p a}$ is determined by the primary transmitter and $W_{v a}$ is determined by the victim receiver.

From the moment generating function $M_{I}(t)$, the expectation of the aggregate interference can be obtained as follows:

$$
\begin{aligned}
E(I) & =\left.\frac{d M_{I}(t)}{d t}\right|_{t=0} \\
& =\lambda \int_{\mathbb{R}^{2}} E\left[U(x) h(x) 10^{\frac{W_{v a}}{10}} \mid \Omega\right] d x \\
& =\lambda \int_{\mathbb{R}^{2}} h(x) E[U(x) \mid \Omega] \cdot E\left[10^{\frac{W_{v a}}{10}}\right] d x .
\end{aligned}
$$

Here,

$$
E\left[10^{\frac{W_{v a}}{10}}\right]=\int_{-\infty}^{+\infty} 10^{\frac{w}{10}} \cdot f\left(w ; \sigma_{v a}^{2}\right) d w=e^{\omega_{v a}}
$$

where $\omega_{v a} \triangleq \frac{\sigma_{v a}^{2}}{2\left(10 \log _{10} e\right)^{2}}$ and $f\left(w ; \sigma_{v a}^{2}\right)$ denotes the probability density function (pdf) of $W_{v a}$, and

$$
E[U(x) \mid \Omega]=\int_{-\infty}^{\eta-s_{p}+g(\|x\|)} f\left(w ; \sigma_{p a}^{2}\right) d w=\beta(x),
$$


where $\beta(x) \triangleq 1-Q\left(\frac{\eta-s_{p}+g(\|x\|)}{\sigma_{p a}}\right)$ and $f\left(w ; \sigma_{p a}^{2}\right)$ denotes the pdf of $W_{p a}$. Using (15)-(17) we have

$$
E[I]=\lambda e^{\omega_{v a}} \digamma\left(s_{a}, x_{v}\right),
$$

with

$$
\digamma\left(s_{a}, x_{v}\right)=\int_{\mathbb{R}^{2}} \beta(x) h(x) d x .
$$

\section{Power Control based on Aggregate INTERFERENCE}

In this section, we develop a power control scheme based on knowledge of the aggregate interference. As described in [3], for a single secondary transmitter, the maximum interferencefree transmit power (MIFTP) can be defined in terms of the worst-case interference probability:

$$
P_{\text {int }}(a)=\sup _{v} P_{\text {int }}(a, v)=Q\left(\frac{I_{\max }-s_{a}+g\left(d_{a}^{*}\right)}{\sigma_{v a}}\right),
$$

where $d_{a}^{*} \triangleq d_{p, a}-d_{c o v}(p)$ is called the critical distance for the secondary transmitter $a$ with respect to the primary transmitter $p$, and the supremum is taken over all potential victim nodes $v$ such that $d_{p, v} \leq d_{\text {cov }}(p)$. The MIFTP is then given by $s_{a}^{*}=\max \left\{s_{a}: P_{\text {int }}(a) \leq \varepsilon_{\text {int }}\right\}$, and we have

$s_{a}^{*}= \begin{cases}I_{\max }+g\left(d_{a}^{*}\right)-\sigma_{v a} Q^{-1}\left(\varepsilon_{\text {int }}\right), & \text { if } d_{p, a}>d_{c o v}(p), \\ -\infty, & \text { otherwise. }\end{cases}$

However, in the model presented here, multiple secondary transmitters may transmit signals at the same time. The critical distance for the $i$ th secondary transmitter is defined as $d_{a_{i}}^{*} \triangleq$ $d_{p, a_{i}}-d_{\text {cov }}(p)=\left\|x_{i}\right\|-d_{\text {cov }}$. In Fig. 1, the critical distances corresponding to the secondary transmitters $a_{1}, a_{2}$, and $a_{3}$, are given by $d_{a_{1}, v_{1}}, d_{a_{2}, v_{2}}$, and $d_{a_{3}, v_{3}}$, respectively. We assume that each secondary transmitter that has the right to access the network can contribute at most $i_{\max }$ interference to the victim node $v$, such that the sum of $i_{\max }$ over all such secondary transmitters should satisfy $\sum_{i \in \mathcal{A}} U\left(x_{i}\right) i_{\max } \leq I_{\max }$. Then the $i$ th secondary transmitter is permitted to transmit at a power level not exceeding its group MIFTP or G-MIFTP defined as follows:

$s_{a_{i}}^{*}= \begin{cases}i_{\max }+g\left(d_{a_{i}}^{*}\right)-\sigma_{v a} Q^{-1}\left(\varepsilon_{i n t}\right), & \text { if } d_{p, a_{i}}>d_{c o v}(p) \\ -\infty, & \text { otherwise. }\end{cases}$

In this case, the problem of obtaining the G-MIFTP for secondary transmitters reduces to choosing the appropriate value of $i_{\max }$. If each secondary transmitter that has permission to access the network can transmit with G-MIFTP, the mean aggregate interference is given by

$$
E[I]=\lambda \cdot 10^{\frac{i_{\max }-\sigma_{v a} Q^{-1}\left(\varepsilon_{i n t}\right)}{10}} e^{\omega_{v a}} \digamma\left(x_{v}\right),
$$

with

$$
\begin{aligned}
\digamma\left(x_{v}\right) & =\int_{\mathbb{R}^{2}} \beta(x) 10^{\frac{g\left(\|x\|-d_{c o v}\right)-g\left(\left\|x-x_{v}\right\|\right)}{10}} d x \\
& =\int_{\mathbb{R}^{2}} \beta(x)\left(\frac{\|x\|-d_{\text {cov }}}{\left\|x-x_{v}\right\|}\right)^{\kappa} d x .
\end{aligned}
$$

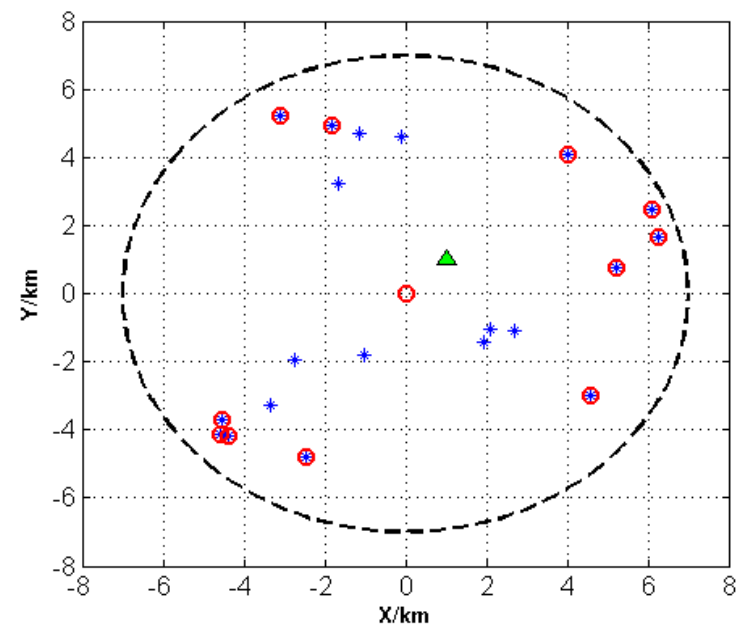

Fig. 2. Simulation scenario.

From the moment generating function of the aggregate interference, we can also obtain $E\left[I^{2}\right]$, and then the variance of the aggregate interference can be obtained as follows:

$$
\operatorname{Var}(I)=\lambda \cdot 10^{\frac{i_{\max }-\sigma_{v a} Q^{-1}\left(\varepsilon_{i n t}\right)}{5}} e^{\frac{\omega_{v a}}{4}} \Gamma\left(x_{v}\right)
$$

with

$$
\begin{aligned}
\Gamma\left(x_{v}\right) & =\int_{\mathbb{R}^{2}} \beta(x) 10^{\frac{g\left(\|x\|-d_{\text {cov }}\right)-g\left(\left\|x-x_{v}\right\|\right)}{5}} d x \\
& =\int_{\mathbb{R}^{2}} \beta(x)\left(\frac{\|x\|-d_{\text {cov }}}{\left\|x-x_{v}\right\|}\right)^{2 \kappa} d x .
\end{aligned}
$$

For a normal random variable $X \sim N(\mu, \sigma)$, the probability that the value of the variable $X$ lies outside of the range $[\mu-$ $2 \sigma, \mu+2 \sigma]$ is very low. Although the exact distribution cannot be obtained, we have the mean and variance of the aggregate interference. Based on this principle, in order to ensure that the aggregate interference from all of the active secondary transmitters is below $I_{\max }$ with high probability, we propose the following equation:

$$
E[I]+n \sigma=10^{I_{\max } / 10},
$$

where $n$ is chosen according to the requirements of the system. From the above equation and (22), given the value of $I_{\max }$, we can obtain an approximate value for $i_{\max }$. In this way, each active secondary transmitter may transmit with a power level up to its G-MIFTP, given by (21), without violating the given interference tolerance of the primary system.

\section{Numerical Results}

In this section, we present numerical results to demonstrate the accuracy of our theoretical results and the performance of our proposed power control algorithm. We choose our simulation scenario as shown in Fig. 2. The area of interest is a disk with radius $R=7 \mathrm{~km}$ and the primary transmitter is located at the center, $(0,0) \mathrm{km}$. The victim node is located at $(1,1) \mathrm{km}$, denoted by a triangle. The stars represent all the secondary transmitters having the opportunity to transmit in the area, and the stars enclosed in circles represent the secondary 


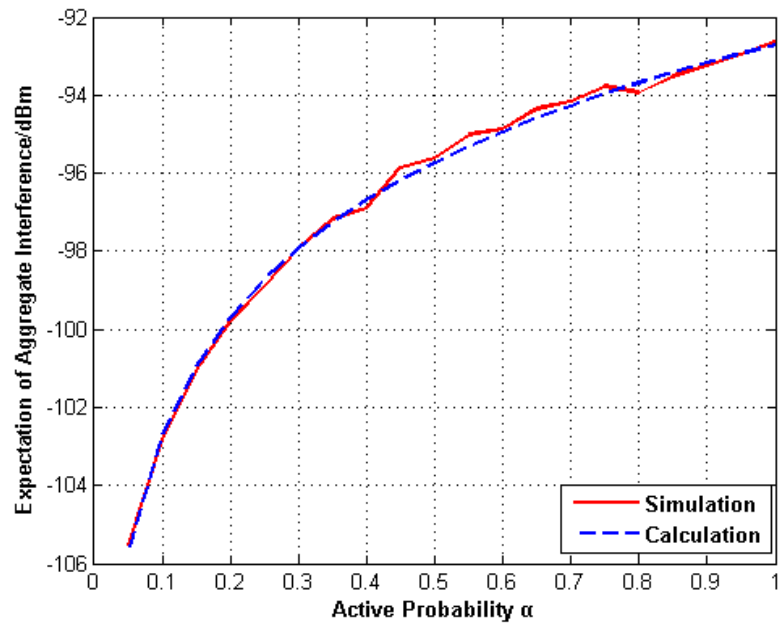

Fig. 3. Expectation of aggregate interference for fixed power $s_{a}$.

transmitters that are transmitting in the same spectrum band as the primary transmitter. The Poisson point process intensity is given by $\Lambda=1.0 \times 10^{-6}$, while the active probability $\alpha$ is a parameter that is varied in our simulation experiments. The path loss factor is given by $\kappa=4$.

\section{A. Fixed transmission power $s_{a}$}

We first consider the case where each secondary node transmits at a fixed power level $s_{a}$. The fixed transmission power for the secondary transmitter is given as $s_{a}=40 \mathrm{dBm}$, and all the other relevant parameters are as follows: $s_{p}=40 \mathrm{dBm}$, $\sigma_{v a}=2 \mathrm{~dB}, \sigma_{p a}=2 \mathrm{~dB}, \eta=-110 \mathrm{dBm}$. We can calculate the expectation of the aggregate interference for the fixed transmission power by using (18). In order to verify this result, we ran simulations with the active probability $\alpha$ ranging from 0.1 to 1.0. Fig. 3 shows that the calculation matches the simulation result very well. When $\alpha$ increases, the mean number of transmitting secondary transmitters increases correspondingly, and the expectation of the aggregate interference also increases.

\section{B. Transmission with G-MIFTP}

Next, we assume that each active secondary node can transmit up to a power level given by its G-MIFTP. The aggregate interference threshold is given by $I_{\max }=-100 \mathrm{dBm}$, and we choose $n=2$ to calculate $i_{\max }$ for each secondary transmitter. The other relevant parameters are as follows: $s_{p}=40 \mathrm{dBm}$, $\sigma_{v a}=2 \mathrm{~dB}, \sigma_{p a}=2 \mathrm{~dB}, \eta=-110 \mathrm{dBm}, \varepsilon_{\text {int }}=0.01$, $\varepsilon_{\text {cov }}=0.05, r_{\min }=-90 \mathrm{dBm}$. Fig. 4 shows that the expected aggregate interference calculated for G-MIFTP using (22) matches the simulation result very well. Moreover, with the power control scheme based on (24), the expected aggregate interference can always be maintained under the threshold $I_{\max }$.

Next, we pick up the active probability $\alpha=0.05$ to further investigate the performance of the system. The power control scheme ensures that the expectation of the aggregate interference, $E[I]$, is below $I_{\max }$. Through simulations, we find that

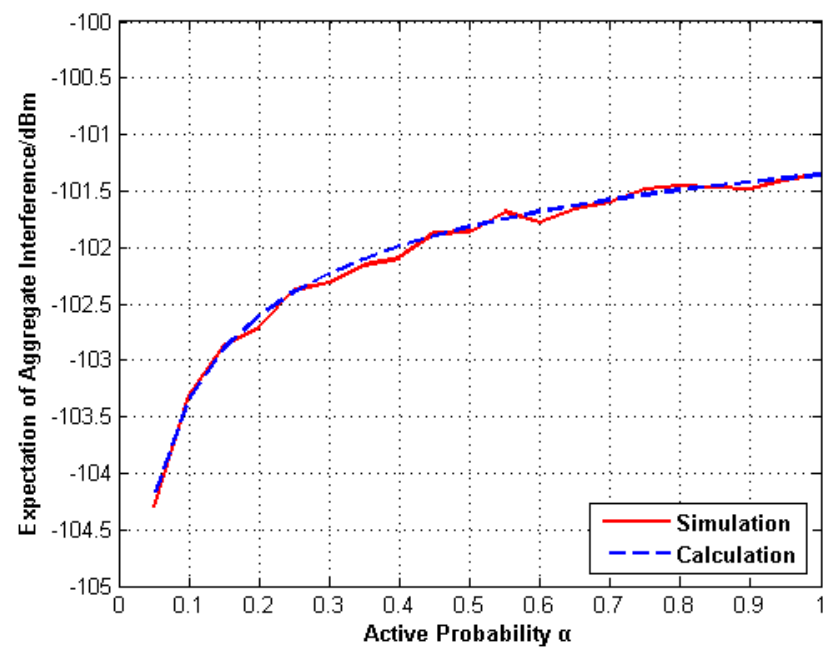

Fig. 4. Expectation of aggregate interference for G-MIFTP model.

the probability that the expected aggregate interference lies outside of the range $[E[I]-2 \sigma, E[I]+2 \sigma]$ is about $6 \%$. If we wanted this probability to be even smaller, we could choose a larger value of $n$ for the system.

Finally, we compare the achievable capacities corresponding to using the G-MIFTP model vs. the MIFTP model. The achievable capacity for the $i$ th secondary transmitter-receiver pair is given by [17]:

$$
C_{i}=B \cdot E\left\{\log _{2}\left(1+\frac{\operatorname{MIFTP}_{i}\left(d_{i} / d_{0}\right)^{-\kappa} W}{N_{0} B}\left|H_{i}\right|^{2}\right)\right\},
$$

where the expectation $E[\cdot]$ is taken with respect to the shadowing noise $W$, Rayleigh fading coefficients $H_{i}$ with $E\left\{\left|H_{i}^{2}\right|\right\}=1$, and the transmitter-receiver distance $d_{i}$. In the simulation, we set $d_{i}=1 \mathrm{~km}$ and $d_{0}=1 \mathrm{~m}$. Fig. 5 shows the capacity improvement achieved by using G-MIFTP rather than MIFTP. The power control scheme based on the GMIFTP outperforms that based on the MIFTP, since multiple secondary nodes are allowed to transmit at the same time.

\section{CONCLUSION}

We obtained an expression for the expectation of the aggregate interference for a cognitive radio network where the secondary transmitters are scattered according to a Poisson point process. Based on knowledge of the aggregate interference, we proposed the G-MIFTP model for power control and found that it resulted in significantly higher achievable capacity for the secondary system by accommodating multiple simultaneous secondary transmissions. The proposed power control scheme ensures that the interference to the primary receiver is kept under a certain threshold; however, the interference between secondary transmitter-receiver pairs was not taken into account. In addition, to apply our results in a real network, it would be of interest to estimate the intensity of the Poisson point process in a given cognitive radio network. We are currently pursuing these and related research issues. 


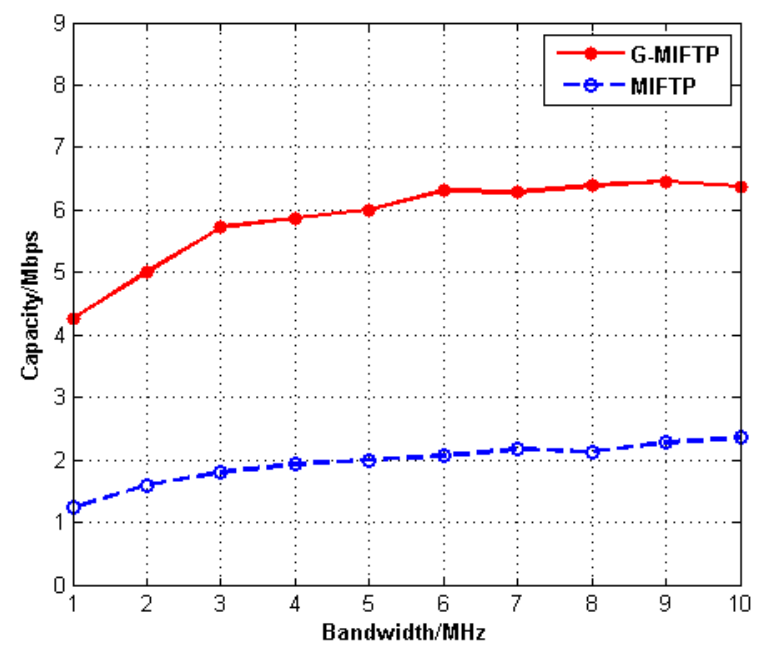

Fig. 5. Achievable capacity under the MIFTP and G-MIFTP models, $\alpha=$ 0.05 .

\section{REFERENCES}

[1] FCC, "Spectrum policy task force," ET Docket 02-135, Nov. 2002.

[2] M. A. McHenry, "NSF spectrum occupancy measurements project summary," Shared Spectrum Company, Tech. Rep., Aug. 2005.

[3] B. L. Mark and A. O. Nasif, "Estimation of maximum interference-free transmit power level for opportunistic spectrum access," IEEE Trans. on Wireless Communications, vol. 8, no. 5, pp. 2505-2513, May 2009.

[4] A. Rabbachin, T. Q. Quek, and H. Shin, "Cognitive network interference," IEEE Trans. on Selected Areas in Communications, vol. 29, no. 2, pp. 480-493, Feb. 2011.

[5] T. V. Nguyen and F. Baccelli, "A stochastic geometry model for cognitive radio networks," The Computer Journal, vol. 55, no. 5, pp. 534-552, 2012.

[6] Y. Chen, G. Yu, and Z. Zhang, "On cognitive radio networks with opportunistic power control strategies in fading channels," IEEE Trans. on Wireless Communications, vol. 7, no. 7, pp. 2752-2761, July 2008.

[7] H. Islam, Y.-C. Liang, and A. Hoang, "Joint power control and beamforming for cognitive radio networks," IEEE Trans. on Wireless Communications, vol. 7, no. 7, pp. 2415-2419, July 2008.

[8] S. Srinivasa and S. A. Jafar, "Soft sensing and optimal power control for cognitive radio," IEEE Trans. on Wireless Communications, vol. 9, no. 12, pp. 3638-3649, Dec. 2010.

[9] A. E. Leu, M. McHenry, and B. L. Mark, "Modeling and analysis of interference in Listen-Before-Talk spectrum access schemes," Int. J. Network Mgmt., vol. 16, pp. 131-141, 2006.

[10] A. E. Leu, B. L. Mark, and M. A. McHenry, "A framework for cognitive WiMAX with frequency agility," Proc. IEEE, Special Issue on Cognitive Radio, vol. 97, no. 4, pp. 755-773, Apr. 2009.

[11] F. Baccelli and B. Blaszczyszyn, "Stochastic geometry and wireless networks, volume 1: Theory," Foundation and Trends in Networking, vol. 3, no. 3-4, pp. 249-449, 2009.

[12] J. W. Mark and W. Zhuang, Wireless Communications and Networking. Pearson Education, Inc., 2003.

[13] M. N. Lustgarten and J. A. Madsen, "An Empirical Propagation Model (EPM-73)," IEEE Trans. on Electromagnetic Compatibility, vol. 19, Aug. 1977.

[14] M. Gudmundson, "Correlation model for shadow fading in mobile radio systems," Electron. Lett., vol. 47, pp. 2145-2146, Nov. 1991.

[15] H. Kobayashi, B. L. Mark, and W. Turin, Probability, Random Processes, and Statistical Analysis. Cambridge, UK: Cambridge University Press, 2012.

[16] D. Stoyan, W. S. Kendall, and J. Mecke, Stochastic Geometry and its Applications, 2nd ed. New York: Wiley, 1996.

[17] T. Do and B. L. Mark, "Joint spatial-temporal spectrum sensing for cognitive radio networks," IEEE Trans. on Vehicular Technology, vol. 59, no. 7, pp. 3480-3490, Sept. 2010. 